\title{
MONZÓN SUDAMERICANO: LA INTEGRACIÓN DE LA CIRCULACIÓN AMAZÓNICA Y ALTIPLÁNICA Y LAS VARIABILIDADES CLIMÁTICAS DEL ALTIPLANO ANDINO CHILENO*
}

\author{
THE SOUTH AMERICAN MONSOON: INTEGRATION OF BOTH AMAZONIC \\ AND ALTIPLANIC CIRCULATIONS AND CLIMATIC VARIABILITIES \\ OF THE CHILEAN ANDEAN PLATEAU
}

Magaly Mendonça ${ }^{\dagger}$

\begin{abstract}
Este artículo presenta, a partir de la consideración de los datos de precipitaciones mensuales y anuales, un análisis de la integración de la circulación amazónica y altiplánica que vincula al centro del continente sudamericano con los Andes centrales. Bajo ciertas circunstancias atmosféricas, masas de aire de la Amazonia pueden alcanzar hasta el altiplano que bordea por el oriente el desierto de Atacama respondiendo a los factores que activan la actuación del monzón amazónico o sudamericano en el verano: convección termal, posición de la alta de Bolivia, desplazamiento de la corriente de Chorro subtropical, comportamiento de la baja del Chaco, todo ello acoplado en forma significativa con eventos ENSO, oscilación decadal del Pacífico y convergencia antártica. Las relaciones geográficas que se observan requieren un análisis espacial integrado de la región sudamericana, que supere las ficticias divisiones nacionales de los climas, sustituyéndola por análisis que contribuyan a su predicción y mejoramiento de la calidad de vida de los habitantes enfrentados a las variabilidades y cambios climáticos.
\end{abstract}

Palabras claves: Monzón sudamericano, variabilidad de las precipitaciones, circulación atmosférica.

This article presents an analysis of the integration of the Amazonian and altiplanic circulation that links the center of the South American continent with the central Andes, based on the consideration of monthly and annual precipitation data. Under certain atmospheric conditions, Amazonian air masses can reach the plateau bordering the Atacama Desert in the east, responding to the factors that activate the action of the Amazonian or South American Monsoon in the summer months: thermal convection, position of the High of Bolivia, displacement of the Subtropical Jet Stream, behavior of the Lower Chaco, all significantly coupled with ENSO events, Pacific Decadal Oscillation and Antarctic Convergence. The geographic relationships that are observed require an integrated spatial analysis of the South American region that surpasses the fictitious national divisions of climates, replacing it with analyzes that contribute to its prediction and improvement of the quality of life of the inhabitants facing climate variabilities and changess.

Key words: South American Monsoon, rainfall variability, atmospheric circulation.

Este artículo presenta, a partir de la consideración de los datos de precipitaciones mensuales y anuales, un análisis de la integración de la circulación amazónica y altiplánica que vincula al centro del continente sudamericano con los Andes centrales. En todo Chile la alta cordillera de los Andes representa una frontera para la circulación atmosférica, separando casi completamente el sector Este o continental sudamericano del oeste o Pacífico, donde se localiza el desierto de Atacama, uno de los más áridos del mundo. Bajo ciertas circunstancias atmosféricas, masas de aire de la Amazonia pueden alcanzar hasta el altiplano que bordea por el oriente el desierto de Atacama, mientras que en la mayor parte del país esta cordillera, elevada y maciza, anula la influencia atlántica. Las lluvias en el altiplano andino se concentran en verano (diciembre-marzo) y se registran irregularmente sobre los $3.000 \mathrm{~m}$ de altura. En verano la corriente en chorro subtropical, que domina la circulación con una componente del oeste, tiene menor intensidad y se localiza más hacia el sur, permitiendo la actuación en las capas troposféricas cercanas a la superficie altiplánica de una débil componente del este y la advección episódica de aire húmedo desde la cuenca amazónica. Aceituno (1996) propone un esquema de circulación regional que considera la advección de vapor de agua desde la cuenca amazónica, centrada en los meses

\footnotetext{
* Proyecto FONDECYT No 1150701.

$\dagger \quad$ En el contexto de los artículos de Geografía que se presentan en este número, Diálogo Andino tiene el honor de publicar el trabajo de la Dra. Magaly Mendonça quien ya no nos acompaña. La Dra. Mendonça, fue Académica de la Universidad Federal de Santa Catarina (Florianópolis, Brasil) y se desempeñaba en el Departamento de Geociencias.
} 
de verano. De esta manera, la temporada de lluvias viene después del máximo forzamiento radiactivo de la superficie altiplánica, que ocurre en la transición primavera-verano, demostrando con ello que este no es suficiente para el desarrollo de la nubosidad convectiva. En este sentido una explicación para la temporada de lluvia en el altiplano andino se asocia a la activación del monzón sudamericano.

\section{Metodología}

Respecto de los climas del altiplano andino se analizarán datos de las regiones chilenas de Arica-Parinacota y Taparacá, localizadas entre las latitudes $18^{\circ}$ y $21^{\circ} \mathrm{S}$-abarcando desde el límite norte de Chile con Perú hasta el salar de Coposa-. Para la interpretación de las causas de las lluvias se consideró el estudio de dos transectos latitudinales que se extienden, el primero mediante la sección norte (regiones de Arica-Parinacota) y el segundo en la sección sur, correspondiente a la región de Tarapacá, desde la costa hasta las cuencas altiplánicas de Caquena y Cancosa, ambas localizadas sobre los 4000 msnm (Figura 1). Los gráficos utilizados son compilados de Romero et al., 2011, donde los autores discuten las características climáticas del norte del altiplano chileno. También fueron consultadas otras bases bibliográficas como Aceituno (1990 y 1996), entre otros. Al mismo tiempo se agregan resultados interpretativos de datos de temperatura y precipitación pertenecientes a la red meteorológica de Bolivia, analizados por Seiler et al.
(2013), considerando índices de cambios climáticos y variabilidades de baja frecuencia, así como la Oscilación Decenal del Pacífico (ODP o PDO en inglés), Fenómeno El Niño Oscilación Sur (ENOS, ENSO en inglés) y Oscilación Antártica (AAO, en inglés). Adicionalmente se hacen referencias acerca de trabajos realizados en Brasil que abordan algunas de esas variabilidades.

\section{Los contextos climáticos}

Las condiciones climáticas del altiplano andino son básicamente controladas por la altitud (3.700 msnm, en promedio) y latitud (en la zona intertropical). Las temperaturas son considerablemente inferiores a las registradas a nivel del mar o en las planicies del este y las extremas diarias están parcialmente determinadas por las variaciones estacionales de nubosidad y del contenido de vapor de agua en la atmosfera. Aceituno (1996) muestra que el contenido de humedad atmosférica en la localidad de Visviri (próxima el hito tripartito de la frontera Chile-Perú-Bolivia) es particularmente reducido durante el invierno $\left(3 \mathrm{gr} \mathrm{Kg}^{-1}\right)$ cuando predomina una circulación del oeste, aumentando más que al doble $\left(7 \mathrm{gr} \mathrm{Kg}^{-1}\right)$ en algunas horas durante el verano, pudiendo alcanzar valores superiores $\left(9 \mathrm{gr} \mathrm{Kg}^{-1}\right)$ en periodos de advección de aire húmedo desde el este.

Las lluvias ocurren de diciembre a marzo (Figura 1) como resultado de la intensa actividad convectiva de la región tropical del continente, de la que hacen parte los Andes Centrales y que

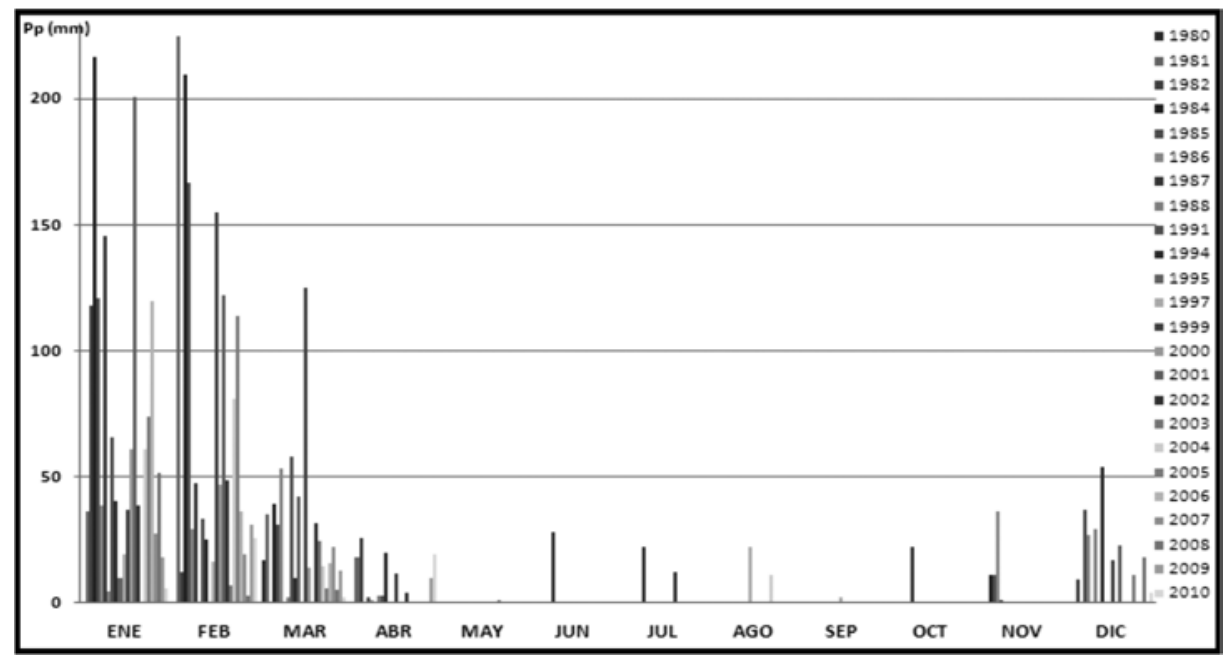

Figura 1. Totales mensuales de precipitación, estación Caquena.

Fuente: Dirección General de Aguas (DGA) in Romero et al. (2011). 
en verano alcanza su mayor intensidad y máximo desplazamiento hacia el sur (Aceituno, 1996). Los registros de precipitaciones en otras estaciones del año son escasos y se asocian a perturbaciones frontales provenientes desde el Pacífico, cuyo impacto alcanza el sector sur occidental del Altiplano (Viulle, 1996).

La distribución espacial de las precipitaciones de la región norte del desierto de Atacama demuestra que estaciones ubicadas a menor altura, como es el caso de Arica (58 msnm), registran pocos milímetros de lluvias, pero en las estaciones de mayor altura, los montos anuales pueden superar los 700 $\mathrm{mm}$ (Figura 2).

En la región de Tarapacá se observa una reducción en los montos de las precipitaciones en la medida que aumenta la latitud, pero al igual que en el caso anterior, solo en el caso de Cancosa, que se encuentra sobre los $3.000 \mathrm{msnm}$, se registra un promedio de 150 milímetros anuales, en cuanto las estaciones restantes poseen montos inferiores a los 80 milímetros (Figura 3) (Romero et al., 2011).

Las lluvias en el altiplano chileno, además de su concentración en verano, alcanzan valores máximos en el borde occidental que superan el $90 \%$ del total anual, demostrando una naturaleza episódica. Ocurren principalmente después del mediodía, asociadas a intensos chubascos caracterizados por nubosidad convectiva de fuerte desarrollo vertical y gran actividad eléctrica. El inicio y mantenimiento de la nubosidad convectiva depende del forzamiento radiactivo y del relieve local, pero los períodos

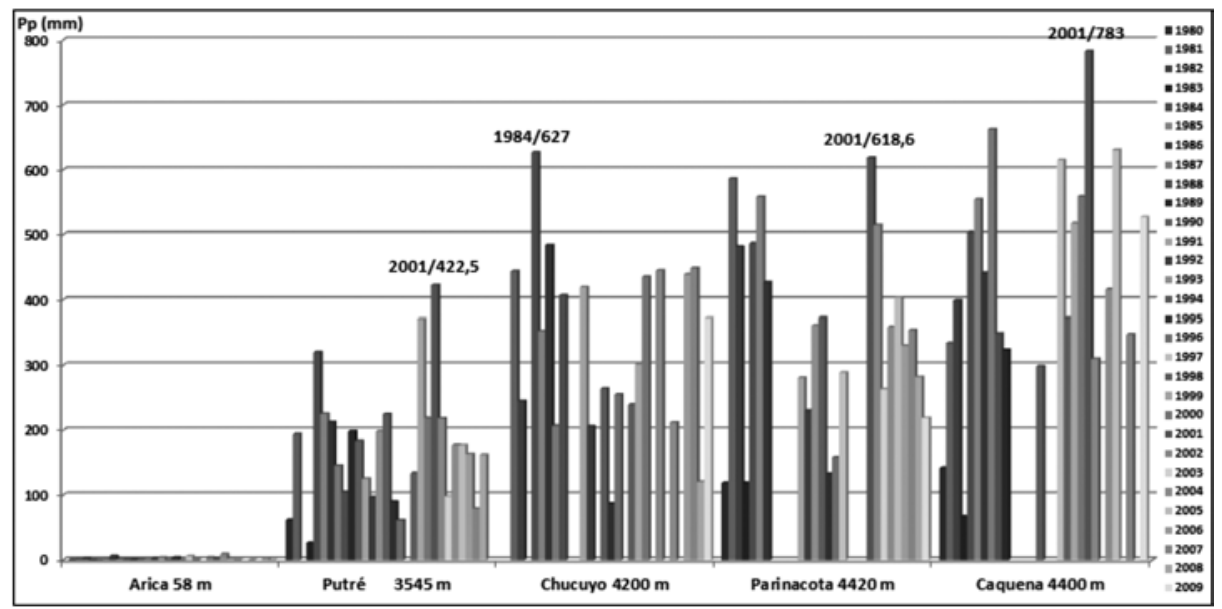

Figura 2. Precipitación total anual a lo largo del transecto latitudinal de la región de Arica y Parinacota. Fuente: Romero et al., (2011).

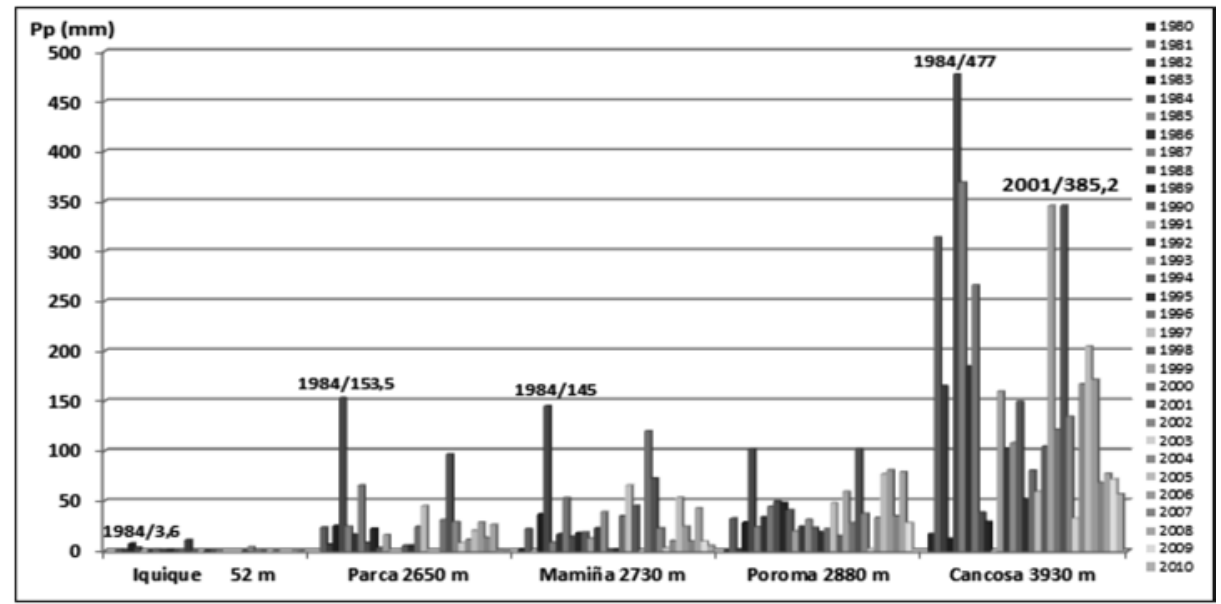

Figura 3. Precipitación total anual a lo largo del transecto latitudinal de la región de Tarapacá.

Fuente: Romero et al., 2011. 
húmedos (secos) en el sector norte (aproximadamente $\left.17^{\circ} \mathrm{S}\right)$ se caracterizan por una alta da Bolivia (AB, Figura 4) anormalmente intensa (débil) y desplazada hacia el sur (norte) (Aceituno y Montecinos, 1993).

En Bolivia, las precipitaciones varían entre 250$2.000 \mathrm{~mm} /$ año debido el relieve, siendo crecientes hacia las tierras bajas.Las precipitaciones estacionales oscilan entre 50 y $250 \mathrm{~mm}$ mensuales en verano y entre 0 y $60 \mathrm{~mm}$ en el invierno. Días secos consecutivos también aumentan desde las tierras bajas hacia el Altiplano, yendo desde menos de 1 mes en las tierras bajas del noroeste hasta 5 meses en el Altiplano suroeste. Días consecutivos húmedos varían entre 5 y $8 \mathrm{~mm}$ con valores más altos en las tierras bajas del norte y menores en las tierras bajas del sur y el Altiplano. Las precipitaciones alcanzan intensidades de 6 a $18 \mathrm{~mm}$ (días húmedos) y durante intervalos de días muy húmedos, fluctuando sumas entre 50 y $400 \mathrm{~mm} / \mathrm{año}$ (Seiler et al., 2013). La temporada de lluvias pronunciadas conduce a inundaciones periódicas de enero a marzo en las sabanas de las tierras bajas (Bourrel et al. 2009). Sin embargo, las inundaciones en los Andes también son posibles, especialmente en las zonas de captación del lago Titicaca y Poopó (PNUD 2011). Las sequías tienen lugar principalmente de junio y agosto en las tierras bajas del sur y en la parte suroeste del Altiplano

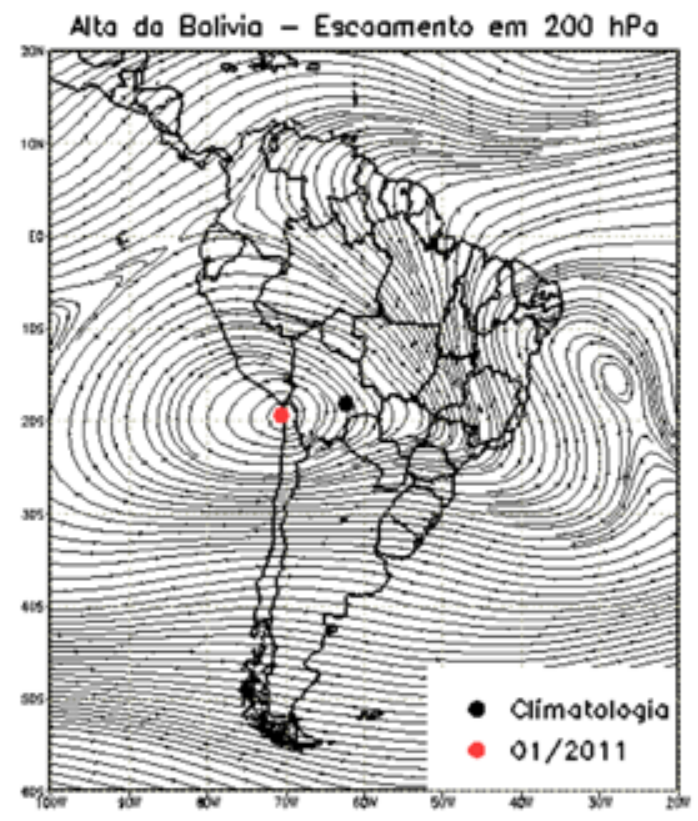

Figura 4. Posición promedio climatológica de la circulación de la alta troposfera en ENERO/2011.

Fuente: INPE/CPTEC.
(CONARADE, 2010). El verano más cálido (DJF) y el invierno más frío (JJA) coinciden con la estación húmeda y seca, respectivamente.

La concentración de la precipitación en verano, su significativa variabilidad espacial caracterizada por un aumento de montos hacia el norte y hacia el este, demuestra la fuerte dependencia del aire húmedo proveniente del sector amazónico. El desplazamiento de la faja convectiva hacia al sur empieza con el equinoccio de primavera en septiembre, meses antes de la temporada de lluvias, y alcanza montos aún bajos en diciembre. Así, la mayor advección del aire del este parece hacer parte de un esquema de circulación más amplio (Aceituno, 1996) que puede ser explicado por el sistema de monzón de América del Sur. De este sistema participan centros de acción de superficie $\mathrm{y}$ altitud, semifijos y estacionales.

\section{La estructura y dinámica del monzón sudamericano}

El sistema del monzón sudamericano comprende una compleja y dinámica estructura espacial integrada principalmente por el cinturón de la Alta Subtropical del Atlántico Norte (ASAN), la Zona de Convergencia Intertropical (ZCIT), la Alta Subtropical del Pacífico Sur (ASPS), Baja Térmica del Chaco (BCH), Zona de Convergencia del Atlántico Sur (ZCAS) y los frentes atmosféricos del Sur (FS), como se observa en la imagen satelital captada por NOAA (Administración del Océano y Atmósfera de los Estados Unidos) y procesada por el Centro de Investigaciones del Tiempo y Estudios Climáticos (CPTEC en portugués) del Instituto Nacional de Investigaciones Espaciales (INPE en portugués) de Brasil, para el 13 de enero de 2010 a las 18 horas (Figura 5).

La caracterización de la circulación del monzón consiste en reversiones estacionales en la dirección de los vientos, que causan veranos lluviosos e inviernos secos (Morany Morgan, 1986), lo que solo se verifica en la región Centro Oeste del Brasil y en el Altiplano andino, y no en el resto de América del Sur.

El desarrollo del sistema del monzón se inicia durante la primavera, debido al aumento de la convección profunda sobre el noroeste de la cuenca Amazónica, que avanza en dirección al sureste de Brasil en noviembre cuando ocurre un cambio en la dirección de los flujos de aire del oeste a uno del 


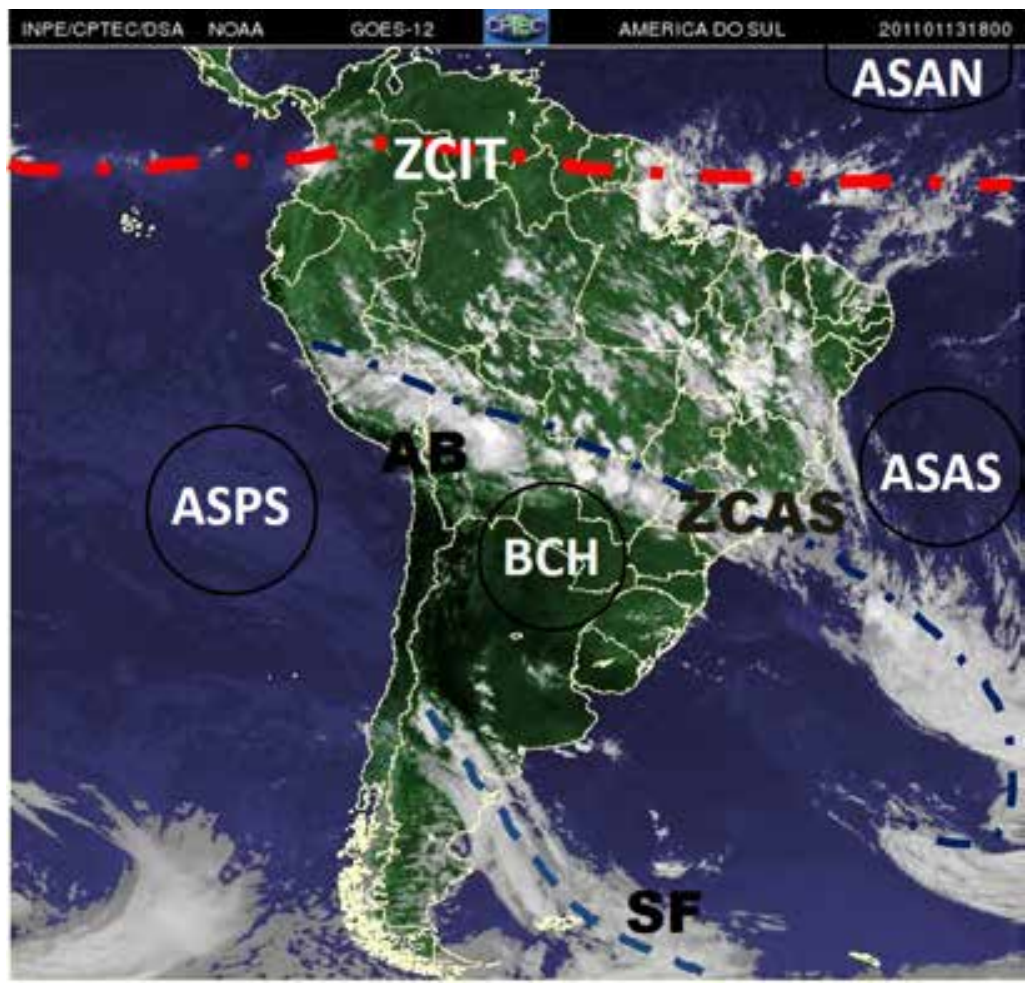

Figura 5: Sistema de monzón de América del Sur.

Fuente: Instituto Nacional de Investigaciones Espaciales (INPE) 2010.

este a los $200 \mathrm{hPa}$ cerca de los $20^{\circ} \mathrm{S}$ (Gan, Rodrigues y Rao, 2009). El inicio, basado en varios autores y diferentes métodos y técnicas, es identificado en la primera quincena de octubre, el más temprano, o en la primera quincena de noviembre el más tardío. En esa variabilidad parece influir el fenómeno ENOS (Marengo et al., 2012).

En octubre ya se puede identificar, en la alta troposfera, la alta de Bolivia (AB, Figura 6) ubicada sobre el Perú y oeste de Amazonia y una depresión en línea localizada sobre el Atlántico sur tropical y subtropical, cerca de la costa del noreste del Brasil. La $\mathrm{AB}$ es una circulación anticiclónica de gran escala que ocurre en la troposfera superior resultante del aumento de la convección continental en la Amazonía, relacionada con el avance meridional de la Zona de Convergencia Intertropical (ZCIT), que en conjunto con la Corriente del Jet Subtropical atraen más hacia el sur el cinturón ecuatorial de los vientos del este (Aceiutuno, 1996).

Entre la primavera y el verano los flujos de noreste de la Alta Subtropical del Atlántico Norte (ASAN) penetran en la región tropical del continente, y en parte por efecto de la cordillera de los Andes, convergen hacia la llanura central de América del Sur, donde se encuentra la Baja del Chaco (Gan, Rodrigues y Rao, 2009). La Baja Térmica del Chaco (BCH) se activa en superficie por el calentamiento convectivo continental aumentando el gradiente de presión con el Atlántico, generando desde la Alta Subtropical del Atlántico Sur (ASAS) flujos lentos del este que desplazándose en superficie llegan a los Andes. El cambio de dirección de los vientos también ocurre concomitantemente con el desplazamiento de la $\mathrm{AB}$ que en enero ya se posiciona más al sur, entre Bolivia y el Mato Grosso del Sur (Alves, 2009).

Las estaciones secas y lluviosas son caracterizadas por la inversión de los vientos zonales al inicio y término de las mismas: los vientos son del este (oeste) en los bajos (altos) niveles atmosféricos durante la estación seca, y del oeste (del este) en la estación lluviosa (Gan, Kousky Y Ropelewsky, 2004). O sea, en la estación húmeda los vientos del noroeste de la Amazonia y los del este del océano convergen hacia la $\mathrm{BCH}$, ascienden en su centro y 


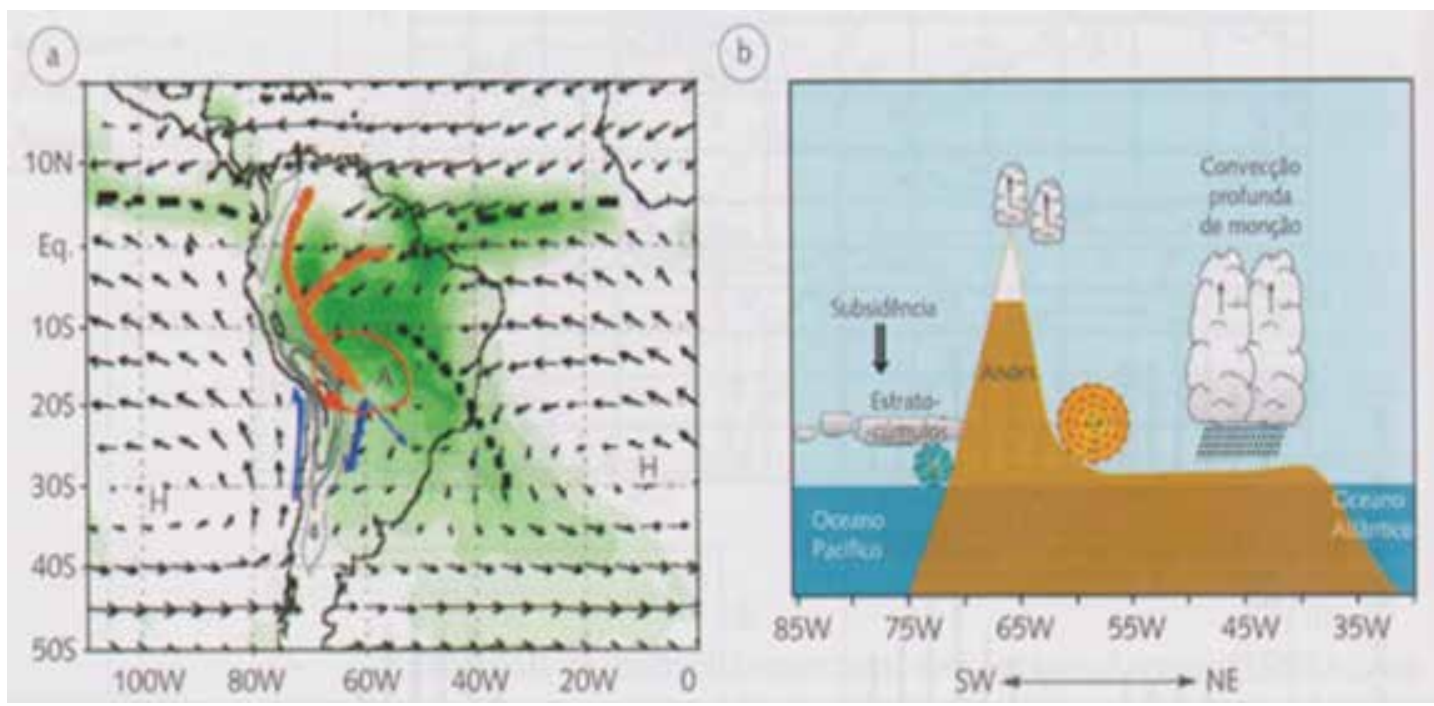

Figura 6. Monzón en América del Sur. a) La parte sombreada indica las precipitaciones y líneas de trazo las zonas de convergencia. Los vectores menores indican el viento en los bajos niveles $(900 \mathrm{hPa}$ ); el vector mayor indica la Corriente de Chorro de los Bajos Niveles; $\mathrm{H}$ indica los anticiclones subtropicales y A la Alta da Bolivia. b) Esquema de la sección vertical del monzón de América del Sur sobre una línea noreste-suroeste de este continente.

Fuente: Compilado de Gan, Rodrigues y Rao, 2009. Mechoso et al., 2005.

divergen en los altos niveles atmosféricos sobre el continente, suministrando el aire húmedo de la convección tropical profunda que ocurre en la planicie continental al este de la cordillera (Romero et al. 2011). En los altos niveles atmosféricos, debido al posicionamiento de la $\mathrm{AB}$ a $200 \mathrm{hPa}$, el vapor de agua del este del continente logra traspasar la barrera de los Andes y transportar humedad hacia el altiplano del norte chileno.

Los máximos totales de precipitaciones entre diciembre y febrero sobre la mayor parte de la región tropical de América del Sur ocurren con el establecimiento de la Zona de Convergencia del Atlántico Sur (ZCAS), que representa al monzón bien configurado. La ZCAS es un escurrimiento convergente de humedad en la baja atmosfera que se presenta como una faja de nubosidad y lluvias con orientación noroeste-sureste, que se extiende desde la Amazonia hacia el sureste de Brasil y también con frecuencia sobre el océano Atlántico Subtropical (Carvalho y Jones, 2009). El flujo transiente de humedad de la Amazonia es importante para la mantención de la ZCAS y su localización es influenciada por la topografía en el centro-este de Brasil, que a su vez influencia notoriamente la posición e intensidad de la AB (Vera et al. 2006a). Aunque la ZCAS sea observada en todos los veranos, hay variabilidad en su organización espacial, intensidad de las lluvias y circulación, que influencian en la ocurrencia de eventos extremos como anegamientos y deslizamientos de tierra. La ausencia de las ZCAS puede representar, al contrario, largos periodos de sequías o de mala distribución de lluvias (Carvalho y Jones, 2009).

La actividad, persistencia y extensión espacial de la ZCAS está asociada a la variación de la Radiación de Ondas Largas (ROL), en regiones con ROL $\leq 200$ $\mathrm{Wm}^{-2}$. La intensidad no depende de su extensión sobre el océano, pues se puede observar intensa y persistente actividad convectiva en el continente sin que ocurra lo mismo sobre el océano. Al contrario, también puede ocurrir intensa convección sobre el océano y sureste brasileño independiente de lo que ocurre en el interior del continente. Jones y Carvalho (2002) muestran que los vientos del oeste de los bajos niveles que cruzan el Ecuador en dirección hacia el sur y terminan en una circulación ciclónica sobre Argentina, están asociados a regiones extensas de convección y lluvias en el Altiplano de Bolivia y norte de América del Sur, caracterizando presencia de ZCAS. En los periodos de ausencia o debilitamiento de las ZCAS (NZCAS) aumenta el número de sistemas convectivos de mesoescala en el noroeste de la Amazonia, lo que muestra que 
el transporte de humedad de los trópicos para los extratrópicos de Sudamérica también es más eficiente durante regímenes de ZCAS.

Otro componente del sistema de monzón es el Chorro de Bajos Niveles (LLJ, en inglés), vientos que tienen velocidad máxima en torno de los 2.000 $\mathrm{m}$ y ocurren en el lado este de la cordillera de los Andes, que bloquea la circulación de los bajos niveles provocando la canalización del aire y transporte de humedad desde la cuenca Amazónica hacia la cuenca de Paraná-Plata, afectando el tiempo y clima de la región este de los Andes. Las variaciones en el transporte de humedad pueden generar condiciones de tiempo severas asociadas a grandes nubes convectivas en la región de salida del Chorro causando tormentas e inundaciones. En verano el suministro de humedad es originado por el paso de los alisios sobre la región de evapotranspiración del bosque, mientras que en invierno proviene principalmente del océano divergente de la Alta Subtropical del Atlántico Sur (ASAS). El transporte de humedad integrado verticalmente es mayor en la región este del Chorro en verano e invierno, pero más intenso en la estación estival por la convergencia de los flujos de los alisios y del Atlántico en el sur del Brasil (Marengo, Ambrizzi y Sores, 2009). Salati et al. (1979) señalan que cerca de $44 \%$ del flujo de vapor de agua que sale de la cuenca Amazónica transportado por los vientos alisios va a condicionar el clima de otras regiones de América del Sur. Mechoso et al. (2005) indican que la humedad transportada desde la Amazonia por el Chorro de Bajos Niveles (LLJ) hasta la porción central da Sudamérica, aumenta la convergencia del flujo de humedad y la precipitación en la región de la Baja del Chaco, mientras en altos niveles la $\mathrm{AB}$ es observada cerca de la región de máxima precipitación, asociada a la subsidencia sobre el Pacífico, donde crea una capa de estratocúmulos, característica semejante a la observada sobre regiones de monzón de otras partes del globo. Así, en ciertos periodos el Chorro (LLJ) también contribuiría para el aumento de la humedad sobre el Altiplano gracias a la divergencia en la AB. Para Garreaud, Vuille y Clement, (2003) la condición episódica de las lluvias demuestra un incremento de la humedad que permite a las corrientes ascendentes locales generar precipitaciones. Los vientos zonales tienen una estrecha relación con los húmedos del este y los secos del oeste. Flujos zonales anómalos han sido asociados con cambios en la posición e intensidad de la Alta de Bolivia con el centro cerca de los $15^{\circ} \mathrm{S}$ y $65^{\circ} \mathrm{W}$ durante el verano, que a su vez se relaciona con el cuasiestacionamiento de las ondas de Rossby que arriban desde las latitudes medias del Pacífico Sur y se amplifican sobre América del Sur subtropical.

Como la precipitación del Altiplano andino tiende a concentrarse en una semana, seguida por un período de sequía de la misma duración, el monzón no es continuo durante toda la estación lluviosa, teniendo una secuencia de fases activas e inactivas, que varían en frecuencia e intensidad de un año para otro. La estación lluviosa en toda Sudamérica puede registrar precipitaciones por sobre o bajo lo normal, que es una característica de su alta variabilidad. Aún más, cuando el monzón está en la fase activa ocurren días con poca o con mucha precipitación. Las tempestades convectivas se organizan a mesoescala, abarcando los Andes Centrales y también a escala topoclimática, diferenciando el comportamiento de las mesetas, laderas y ejes de drenaje fluviales de cada una de las cuencas altiplánicas (Romero et al., 2011). La combinación de circulación de meso y topoescala climática ha desencadenado eventos extremos de lluvia en varias regiones del Brasil (Rio de Janeiro, São Paulo, Minas Gerais, Santa Catarina, Rio Grande do Sul), así como en Chile en las regiones de Arica-Parinacota, Tarapacá y Antofagasta entre 2010 y 2013.

Gan, Rodrigues y Rao (2009) sugieren que los períodos secos pueden estar asociados a la intensificación de las Corrientes de Chorro de los Bajos Niveles (LLJ), así como la fase activa estaría asociada a la actuación de sistemas sinópticos, como sistemas frontales y vórtices ciclónicos en los altos niveles atmosféricos, que embutidos en la Zona de Convección Tropical (ZCT) aumentan el total de las precipitaciones en la región monzónica. La ausencia de los sistemas sinópticos disminuiría el total de precipitaciones, caracterizando la fase inactiva. Sin embargo, hay una variabilidad espacial de la distribución de las lluvias asociada a actuación de los distintos sistemas que afectan el tiempo en Sudamérica. Marengo et al. (2009) señalan una fase de dipolo cuando una ZCAS fortalecida suprime la convección hacia el sur induciendo ondas de calor sobre las regiones subtropicales y otra en que la supresión de la ZCAS incrementa la convección hacia el sur, por el fortalecimiento del Chorro (LLJ), cuyo transporte de humedad de la cuenca Amazónica puede causar eventos extremos de corta duración en las planicies del centro de Argentina. 


\section{Las variabilidades de baja frecuencia}

La extrema variabilidad interanual de las precipitaciones altiplánicas se manifiesta en la ocurrencia de años completamente secos, hasta otros, que en función de la altura, pueden aproximarse a $1.000 \mathrm{~mm}$ anuales. Los sistemas de precipitación pueden ser potencializados por la presencia de la fase fría del ENSO en verano, como por ejemplo ocurrió en 1984 y 2001 cuando se registraron montos totales de precipitaciones cercanos o superiores a 100 milímetros en las estaciones de la región de Taparacá y muy superiores en Arica y Parinacota. Cancosa presentó valores superiores a $350 \mathrm{~mm}$ en los dos eventos La Niña mencionados. En la región de Tarapacá, en las estaciones por sobre los $2.500 \mathrm{msnm}$, los mayores totales fueran registrados en 1984, mientras en Arica y Parinacota ocurrieron en 2001, a pesar que en Chucuyo destácase el total de 1984. La desigual distribución espacial se observa por mayores montos en las estaciones ubicadas en altitudes elevadas más al norte y este de estas regiones.

Existe así una relación entre las anomalías de los vientos locales y el comportamiento del Océano Pacífico. Los vientos del nivel superior son parte de la amplitud de la respuesta atmosférica al patrón espacial de la temperatura superficial del mar (TSM) en el Pacífico Tropical y proporcionan la conexión física entre la forzante oceánica y el clima de los Andes centrales. Garreaud, Vuille y Clement (2003) muestran (Figura 7) una influencia significativa de El Niño Oscilación del Sur (ENSO) en la precipitación registrada en los meses de diciembre, enero y febrero en el Altiplano, lo que es resultado de una pronunciada gradiente de presión entre las latitudes tropicales y subtropicales del sur, consistente con las relaciones este/húmedo y oeste/seco derivadas de la escala sinóptica. Con el enfriamiento de la zona tropical del Pacífico (Fenómeno La Niña) hay un estrechamiento de la troposfera tropical y por tanto un mayor aumento en el flujo del este hacia el Altiplano, en respuesta a las diferencias de presión y temperatura entre las latitudes tropicales y subtropicales del sur, revirtiendo el patrón durante los veranos secos. Por tanto el gradiente de presión es un factor dominante para regular la precipitación en el Altiplano, aspirando la humedad disponible en las tierras bajas tropicales (Garreud, Vuille y Clement, 2003) (Figura 7).

La influencia del ENSO también es observada en el sistema de monzón, resultando en fechas anticipadas y atrasadas para inicios tardíos durante periodos El Niño y adelantados en episodios La Niña para la temporada de lluvias en Brasil (Marengo et al., 2012 y sus referencias). Estos mismos autores señalan cambios interdecadales a mediados de 1970 correspondientes a Oscilaciones del Pacífico (ODP) y Atlántico Norte (NAO), con diferencias menores en la circulación de Hadley y del monzón entre los períodos de 1958-1976 y 1977-1995, así como en la circulación de Walker, que en el segundo

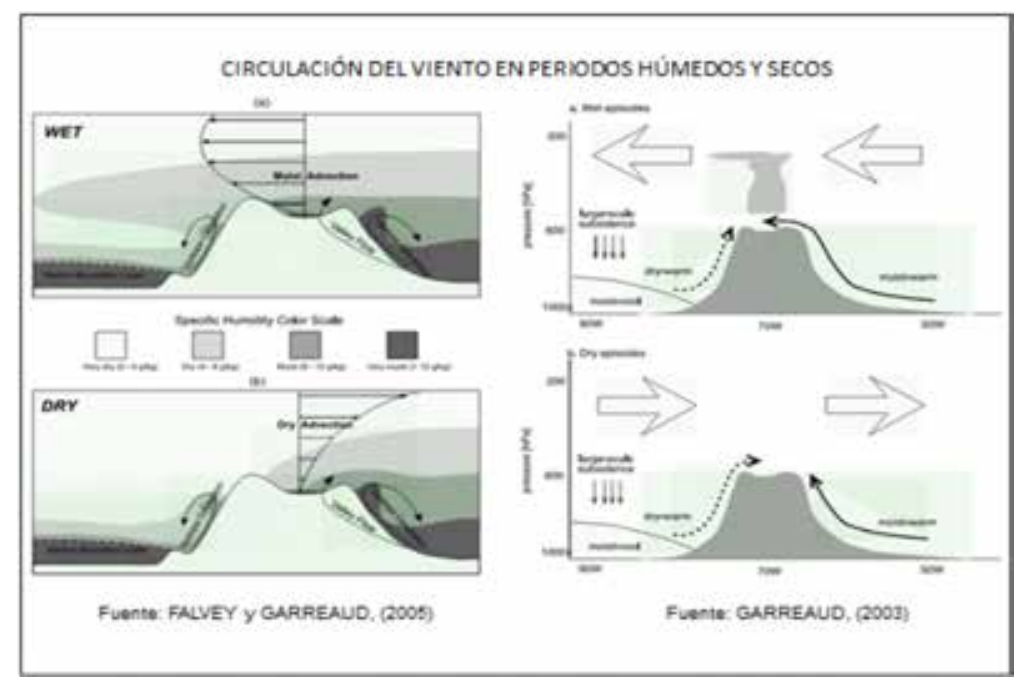

Figura 7: Influencia del ENSO en Sudamérica.

Fuente: Garreaud (2003); Falvey y Garreud (2005). 
período presentó centros de presión más débiles y más distantes hacia el oeste del Pacífico. Otros autores citados también identifican aumento en el flujo de humedad verticalmente integrado sobre Sudamérica después de 1971-72.

Seiler et al. (2013) cuantificaron los impactos de la Oscilación Decadal de Pacífico (ODP), El Niño Oscilación Sur (ENOS), y Oscilación Antártica (AAO) sobre la precipitación media y extrema y detectaron tendencias desde 1960 hasta 2009 en Bolivia, distinguiendo entre las regiones y las estaciones climatológicamente contrastantes. Los autores encontraron que la cantidad total de lluvia, así como el número de eventos extremos, fueron mayores durante fases positivas de ODP, en años El Niño (EN) y La Niña (LN) en las tierras bajas. Durante el verano austral (DJF), EN llevó a condiciones más secas en los Andes, con precipitación más variable. Los totales de precipitación aumentaron desde 1965 hasta 1984 (12\% en DEF y 18\% en JJA) y después disminuyeron ( $-4 \%$ en DEF y $-10 \%$ en JJA), siguiendo aproximadamente el patrón de la ODP. Las tendencias de los extremos climáticos en general corresponden a las tendencias de las medias climáticas. Los autores, sin embargo, son muy cautelosos con sus propias interpretaciones y confianza de los datos.

\section{Consideraciones finales}

La investigación acerca del origen y variabilidad de las lluvias en el Altiplano chileno, porción de un país que no hace frontera con Brasil, es una prueba contundente de que la naturaleza y no solamente la historia une a los pueblos latinoamericanos.

No habría dudas acerca de la ausencia de fronteras en el caso de la atmosfera, pero sí en la consideración de las influencias provenientes del este que avanzan hacia oeste. El Sistema de Monzón, aún poco conocido por los geógrafos, demuestra que mucho más nos une que separa, que traspasar las fronteras políticas del conocimiento es hoy una necesidad.

La expansión urbana experimentada con gran intensidad y poca ciencia en nuestros países revela cada día los innúmeros desastres calificados como naturales, que son bastante selectivos en sus consecuencias sociales. Buscar y encontrar en la naturaleza y sociedad las respuestas para evitar el desastre y distribuir las ganancias ofrecidas por nuestros climas es un desafío que necesita ser enfrentado. Si la naturaleza es capaz de generar medios para la distribución de la humedad, desafiando la propia geografía, debemos ser capaces de generar un ambiente de investigación y discusión académica para encarar y aprender con nuestras buenas y malas experiencias de mitigación del flagelo de los desastres naturales y de la inclusión de nuestros pueblos en la calidad de vida que puede ofrecer la sociedad urbana moderna.

El sistema de monzón y sus componentes demuestran la extrema complejidad de las interacciones naturales, donde el océano, la radiación solar, la vegetación, el releve, entre otros factores, se combinan para determinar la lluvia o la sequía, la distribución de beneficios y riesgos en el espacio y tiempo. Conocer profundamente esos procesos y mecanismos, a partir de la colaboración académica internacional, puede llevarnos a la anticipación de los desastres, al monitoriamiento de los riesgos y al manejo de las potencialidades de las interfaces entre los sitios, la sociedad y atmósfera buscando generar riqueza y calidad de vida.

Profundizar la influencia de cada componente del sistema de monzón en cada porción del suelo latinoamericano, de modo de sacar provecho de las variabilidades anuales, interanuales e interdecadales, a partir del delineamiento de distintos escenarios que administrados en conjunto pudiesen beneficiar la producción alternada de bienes siendo una buena manera de enfrentar las crisis económicas en que vivimos permanentemente. 


\section{Referencias Citadas}

Aceituno, $\mathrm{P}$

1996 Climate elements of the South American Altiplano. Rev. Geofisica-IPGH, 44, 37-55.

Alves, L. M.

2009 Clima da Região Centro-Oeste do Brasil. In: Cavalcanti, I. F. de A.; Ferreira, N. J.; Silva, M. G. A. J.; Dias, M. A. F. da SILVA (orgs) Tempo e Clima no Brasil. São Paulo: Oficina de Textos, pp. 235-24

Bourrel, L.; Phillips, L.; Moreau

2009 The dynamics of floods in the Bolivian Amazon basin. Hydrol. Processes, 23, 3167.

Consejo Nacional de Reducción de Riesgos y Atención de Desastres y Emergencias (CONARADE)

2010 Plan de atención de la emergencia humanitaria y agropecuaria por sequia para el Chaco Boliviano.

Carvalho, L. M. V.; Jones, C.

2009 Zona de Convergência do Atlântico Sul. In: Cavalcanti, I. F. de A.; Ferreira, N. J.; Silva, M. G. A. J. da; Dias, M. A. F. da Silva (orgs). Tempo e Clima no Brasil. São Paulo: Oficina de Textos, pp. 96-109.

Dirección General de Aguas (DGA)

2005 Divisoria de subsubcuencas por regiones. Departamento de Estudios y Planificación, Unidad SIG. Cartografías digitalizadas. Dirección General de Aguas. Santiago, Febrero de 2000, Chile.

Gan, M. A.; Kousky, V. E.; Ropelewsky, C. F.

2004 The South America monsoon circulation and its relationship to rainfall over West-Central Brazil.Journal of Climate, v.17, pp. 47-66.

Gan, M.; Rodrigues, L. C.; Rao, V. B. Monção na América do Sul 2009 In: Cavalcanti, I. F. de A.; Ferreira, N. J.; Silva, M. G. A. J. da; Dias, M. A. F. da SILVA (orgs) Tempo e Clima no Brasil. São Paulo: Oficina de Textos, pp. 297-316.

Garreud, R., Vuille, M.; Clements, A.

2003 The climate of the Altiplano: Observed current conditions and past change mechanisms. Paleo3, 3054, 1-18.

Jones, C.; Carvalho, L. M. V.

2002 Active and break phases in the South American monsoon system. Journal of Climate, v. 15, pp. 905-914.
Marengo, J. A. ; Liebmann, B. ;Grimm, A. M.;Misra, V.; Silva Dias, P. L.;Cavalcanti, I. F. A.;Carvalho, L. M. V.;Berbery, E. H.;Ambrizzi, T.; Vera, C. S.;Saulo, A. C.;Nogues-Paegle,

J.;Zipser, E.; Seth, A.; Alves, L. M.

2012 Review recent developments on the South American monsoon system.International Journal of Climatology. N.32; pp. 1-21.

Marengo, J. A.; Ambrizzi, T.; Soares, W. R. Jato de Baixos Níveis ao longo dos Andes

2009 In: Cavalcanti, I. F. de A.; Ferreira, N. J.; Silva, M. G. A. J. da; Dias, M. A. F. da Silva (orgs) Tempo e Clima no Brasil. São Paulo: Oficina de Textos, pp. 169-180.

Mechoso, C. R.; Robertson, A. W.; Ropelewsky, C.F.; Grimm, A. M. 2005 The American monsoon systems: An introduction. In: Chang, C. P.; Wang, B.; Lau, N.-C. G. (Eds.). The global monsoon system: research and forecast. Genebra: World Meteorological Organization, WMO/TD, n. 1266 (TMRP Rep. n. 70), pp. 197-206.

Melo, A. B. C. de; Cavalcanti, I. F. de A.; Souza, P. P.

2009 Zona de Convergencia Intertropical do Atlántico. In: Cavalcanti, I. F. de A.; Ferreira, N. J.; Silva, M. G. A. J. da; Dias, M. A. F. da Silva (orgs.) Tempo e Clima no Brasil. São Paulo: Oficina de Textos, pp. 25-41.

Moran, J. M.; Morgan, M. D.

1986 Meteorology; The atmosphere and the science of weather. Minneapolis: Burgess Publishing.

Romero, H., Mendonça, M., Méndez, M. y Smith, P.

2013 "Macro y mesoclimas del Altiplano Andino y Desierto de Atacama: Desafíos y estrategias de adaptación social ante su variabilidad". Revista de Geografía Norte Grande, PUC-Chile, volumen 55, pp. 19-41.

Romero, H., Mendonça, M., Méndez, M. y Smith, P.

2011 "Multiescalaridade, Relações Espaciais e Desafios Ecológicos-sociais da Climatologia Sudamericana. O caso do Deserto de Atacama". Revista Brasileira de Climatologia $\mathrm{N}^{\circ}$ 8, 2011. http/ojs.c3sl.ufpr.br/ojs2/index.php/revistaabclima. Fecha: 28/03/2012

Seiler, C.; Hutjes, R. W. A.;Kabat, P.

2013 Climate Variability and Trends in Bolivia.Journal of Applied Meteorology and Climatology v. 52, pp. 130-146. 